pull and the fragments are not ground together. There is immediate relief from pain, due to these causes, after the plaster has been applied; although there is sometimes, for the first twenty-four or forty-eight hours, pain and discomfort due to the pressure of the perineal band across the front part of the thigh and of the thrust upwards against the tuber ischii. The former may be relieved by passing a blunt hook beneath the plaster and pulling the band in front of the thigh upwards or pushing it downwards. The latter yields at once to slight alterations in the position of the limb. If the plaster has not been carried up any great distance above the crest of the ilium, the injured limb may be carried outwards, and so a fresh portion of the soft tissues over the taber ischii receives the pressure. Also abduction of the limb will lessen the force of the upward thrust of the ischial crutch. The skin in this region may also be drawn from side to side twice a day. It is occasionally useful to adduct or abduct the sound limb in order to vary the incidence of pressure.

Whenever possible, and provided that no strips of malleable iron interfere with the passage of $x$ rays to the fracture, it is advisable during the first few days to have an $x$-ray photograph made through the plaster. If it should happen that the fragments are not in good position, the plaster may be taken off and the limb put up again. When the shortening is between 2 and 3 inches, an attempt at an immediate reduction of the total shortening at one sitting may give rise to serious discomfort owing to the resistance of the muscles of the thigh to the upward thrust of the ischial crutch. In these cases I would counsel that at the first attempt the effort should be confined to reducing part of the shortening, say to within an inch, and that in two or three weeks' time the shortening should be further reduced before the plaster is reapplied.

Once the limb is placed

entirely at rest the tendency is for the discomfort to cease gradually and the wounds to close, because all is quiet. One other advantage of this method may be mentioned: not only can the patient be transported a considerable distance without pain, but also, with the limb securely guarded in every direction, he is able to get up at a much earlier date than otherwise, and can go about on crutches.

I give illustrations of $x$-rays (Fig. 9), showing one type of case to which this form of treatment is applicable, and where the shortening, when the first plaster had been removed, was found to be reduced to 1 inch.

\section{RECENT OBSERVATIONS ON THE PATHOLOGY} OF ULCER OF THE STOMACH, WITH INDICATIONS FOR TREATMENT."

\section{[With Special Plate.]}

By CHARLES BOLTON, M.D., D.Sc.Lond., F.R.C.P.Lond.,

PHYSICIAN TO OUT-PATIENTS, ONIVERSITY COLLEGE HOSPITAL CNIVERSITY COLLEGE HOSPITAL MEDICAL SCHOOL.

It has naturally been considered for many years past that the gastric juice played an important part either in the initiation or propagation of gastric ulcer. This idea has, however, been held in a vague kind of way and has not been supported by any very definite evidence with regard to what precise effects this secretion produces and as to

* An adaress delivered before tho Nottingham Medico-Chirurgical Bopciety. what its mode of action really is. The question is of considerable importance in relation to the treatment of gastric ulcer; I therefore propose to discuss the subject, and hope to show that it is the acid of the gastric juice which is the chief damaging factor, and that it acts upon the wall of the stomach, both as an irritant and as a necrotic agent.

Efforts to prove that the ferment is the chief destractive agent and that the gastric juice can digest the wall of the stomach because the latter is deficient in protective antr. ferment have proved unsuccessful, and treatment directed to replace this presumably absent antibody has had no better luck. Moreover, on consideration it seems untikely that such a hypothesis should be correct. If the gastric mucous membrane were deficient in antipepsin the whole or greater part of the membrane should be digested and not merely a circumscribed portion. In no other part of the alimentary canal are ulcers found comparable to gastric and duodenal ulcers. If antiferment is occasionally absent from the stomach it should be now and again absent from the intestine, but the pancreatic juice does not produce lesions comparable in any way to gastric ulcer. Dilute hydrochloric acid is a powerful protoplasmic poison, and, owing to its presence, the gastric juice is alone amongst the digestive juices able to digest connective tissue, and therefore disintegrate foodstuffs and organs whose elements are bound together by that tissue.

Self-digestion of the Stomach BY Normad Gastric Juice.

In many forms of gastric ulcer a definite initial damage to the mucous membrane can easily be demonstrated-for example, in those due to bacterial infection and other causes of necrosis and haemorrhage, also in follicular ulceration. In such cases it is quite easy to understand how the gastric juice can attack the damaged portion of mucous membrane.

In other cases, however, the damage falls short of pro. ducing a visible pathological lesion, but is sufficient to lower the resistance of the mucous membrane to the gastric juice, which attacks the damaged part and com. pletes the death of the tissue. Such damage may be done by poisons circulating in the blood. I was able to demon. strate this principle of self-digestion experimentally some years ago. This was done by producing a gastrotoxic serum active against the gastric mucous membrane, and studying its precise mode of action. Such a' serum is manufactured by immunizing an animal by the repeated injection of the gastric cells of another animal. The immunized animal forms in its blood poisons which, on injection into the body of a similar animal to those whose cells have been injected, give rise to necrosis and ulceration of the mucous membrane of its stomach.

The poison fixes itself to the gastric cells rapidly and produces its effects within a few hours. If the gastric juice be neutralized with an alkali whilst the poison is acting, the mucous membrane is found intact on the death of the animal, whilst the controls with an active gastric secretion show the usual necrosis and ulceration. No microscopic lesion whatever is to be detected in the gastric cells which have been acted on by the poison when the gastric juice is thus put out of action. The cells have, however, been damaged to such an extent that the normal gastric juice is able to attack the wall of the stomach.

The resulting necrotic patches in the mucous membrane are stained black by the action of the hydrochloris acid upon the blood, and soon separate, leaving cleanly punchedout ulcers.

Similar necrosis and ulceration does not occur in the intestine when an enterotoxin is injected, although its mucous membrane is exposed to the active pancreatio juice. 
It is, therefore, to be concluded that the hydrochloric acid of the gastric juice completes the death of the tissue already devitalized by the gastrotoxin, and that this is then digested and removed. Hydrochloric acid of the strength found in normal gastric juice-namely, about 0.2 per cent. - is thus a potential protoplasmic poison, and is able to add its quota to that of a blood poison and become active in its presence.

This is the probable explanation of the frequency with which acute ulcers are found in various toxic and infective conditions of the body.

Increased Destructive Power due to Hyperacidiry.

Hyperacidity of the gastric juice is commonly found in chronic gastric ulcer. The degree of hyperacidity varies considerably. It is very frequently above 0.3 per cent. and sometimes above 0.4 per cent. The highest which has been recorded is 0.5 per cent. Of course, it is only free hydrochloric acid which is able to act as a proto plasmic poison, so that in the early stages of digestion the gastric juice is much less destructive to the mucous mem brane than in the later stages when free hydrochloric acid is present. It is most destructive in cases where there is also hypersecretion, in which condition the secretion of gastric juice continues for varying periods of time after gastric digestion has come to an end. I found experi mentally that when an animal was under the influence of gastrotoxin an increase of the acidity of the gastric juice caused a marked increase in the amount of necrosis and ulceration of the mucous membrane, so that a striking contrast is seen between the stomachs of these auimals with hyperacidity and those of the controls with a normal acidity. This effect is seen with any degree of hyperacidity above the normal, and with strengths of hydrochloric acid which are quite innocuous when administered alone. For example, single doses of hydrochloric acid of any strength up to 0.7 per cent. in the case of small, and 0.9 per cent. in the case of large, guinea-pigs are quite innocuous, and yet a 0.3 per cent. solution will jncrease the necrosis due to gastrotoxin. The effect is progressively more marked as the strength of the acid is increased.

As affording confirmation of the hypothesis that this effect is really due primarily to the poisonous action of the hydrochloric acid and not to any exalted action of the pepsin, I may mention that I found precisely the same result to occur when other mineral acids were employed in dilute solutions which were innocuous alone. We may therefore conclude that the gastric juice owes its destructive power primarily to the hydrochloric acid which is present in it, and that this power progressively increases as the strength of the acid is increased. In the presence of certain blood poisons the normal gastric juice is able to attack the gastric mucous membrane by virtue of the hydrochloric acid which it contains, and the effects produced are much greater when hyporacidity is present.

\section{Effect of Hyperacidity Alone.}

We must now consider the action of various strengths of hydrochloric acid upon the mucous membrane of the healthy stomach.

The effects of a single dose of hydrochloric acid are of no interest to us except from the point of view of the experiments just described, but the effects of repeated doses of such strengths as are found in the condition of hyperacidity in man are of great importance. I have found that repeated doses in the monkey produce two effects: (1) Irritant, (2) necrotic.

\section{The Irritant Effect.}

This is manifested (a) as a more or less diffuse gastritis, (b) by definite local patches of gastritis, (c) by follicular ulceration.

(a) In the case of diffuse gastritis there is found an in. creased amount of mucus in the stomach. In a slight case the mucus secreting cells are swollen and clear and there is slight round-celled infiltration commencing between the glands and most marked near the surface of the mucous membrane. The lymph follicles over and under the muscularis mucosae are a trifle enlarged, and there may be increased vascularity: In advanced cases there is desquamation of the surface epithelium and of the cells of the glands. The interglandular infiltration is very marked, the glands are separated and disappear, leaving a tissue chiefly composed of small round cells and proliferated connective tissue cells, with the remains of glands embedded in it. (Fig. 1.) The bases of the glands are much less affected than the portions nearer the surface and the ducts.

The surface of this tissue becomes re-covered with flat or cubical epithelial cells. In places follicular ulcers are present.

(b) The same process is apparent in the local patches of gastritis, which may be quite sharply marked off from the unaffected portions of the mucous membrane. Some of these arise by extension of the infiltration which spreads from enlarged follicles.

(c) The follicular ulcers arise by enlargement of a lymphatic follicle, which makes its way to the surface through the muscularis mucosae, and by displacing and destroying the gastric glands (Fig. 2). Finally, an ulcer results by removal of the surface epithelium. Such an ulcer to the naked eye has the appearance of a small white elevation in the mucous membrane with a hole in the centre (Fig. 3). These ulcers appear either isolated or surrounded by a condition of gastritis. The continued administration of hydrochloric acid does not deepen the ulcer or make it spread laterally. As a fact, the surface of the ulcer can often be seen to be covered with a single layer of newly formed epithelial cells and healed. There is no doubt that such an enlarged follicle often forms the nucleus of a patch of gastritis, which spreads by the continued administration of hydrochloric acid.

These irritant effects of hydrochloric acid are produced in the monkey by the daily administration of a solution of 0.28 per cent. in strength, and are progressively more marked when stronger solutions are employed. Solutions of 0.5 per cent. in strength, and also stronger ones commonly cause vomiting, so that the effects vary in intensity. The effects likewise depend on the length of time the administration is continued, but quite a considerable amount of gastritis is present after a few doses. The results also vary in individual animals to some extent.

It is remarkable that these results are obtained so easily, because such solutions leave the stomach very rapidly, and the degree of acidity quickly falls. For example, in one experiment $50 \mathrm{c.cm}$. of a 0.39 per cent. solution were administered, and in the space of half an hour later the stomach contained $15 \mathrm{c} . \mathrm{cm}$. of a clear fluid containing only 0.12 per cent. $\mathrm{HCl}$. In the human being the acid has a much longer time in which to act, particularly if hypersecretion or retention of food is present, and there is no reason to suppose that the stomach of man is much less susceptible to the action of $\mathrm{HCl}$ than that of the monkey, since they secrete about the same strength of acid. It seems, therefore, quite certain that hyperacidity, even though not excessive in degree, will give rise to this form of gastritis in man.

Chronic ulcer of the stomach is always associated with a type of gastritis similar to this, and it has more than once been suggested that chronic ulcer may be an advanced form of the follicular ulcer which accompanies this gastritis. It seems to me much more likely that the gastritis and follicular ulceration result from the hyperacidity which so frequently accompanies chronic ulcer. We have seen that the follicular ulcers cannot be transformed into spreading ulcers by continued hyperacidity; and, although commonly found in association with chronic ulcer, it is more likely that they are merely a part of the accompanying gastritis than that they constitute the origin of chronic ulcer.

Gastritis of this form is seen quite apart from chronic ulcer, and probably there are many causes for it. It gives rise to vomiting of blood; and, as I have before given reason to suppose, it is most likely responsible for the vomiting of moderate amounts of blood in young women, which cases have formerly been called "vicarious menstruation," "gastrostaxis," and so on. The follicular ulcers in this gastritis during their formation may open up an artery in the submucous tissue, and cause sudden and profuse haematemesis.

Such acute ulcers may be of various sizes according to circumstances. They commonly heal up, as I have already stated that they do in the monkey, and leave small stellate scars. Such patients may have several attacks of haematemesis at intervals. 
2. The Necrotic Effect.

The necrotic effect of hydrochloric acid is less common than the irritant effect.

The weakest solution which I have found to produce this effect in the monkey is one of 0.38 per cent. strength. A high degree of motor insufficiency was present in this particular case, and it must be regarded as unusual with strengths of acid below 0.4 per. cent.

There is more irregularity in its occurrence, owing to individual variation, than in the case of gastritis.

In the production of this ulceration the ferment in the gastric juice is no doubt also concerned, and spreading acute ulcers are produced by the gradual spread and separation of the necrotic tissue, the $\mathrm{HCl}$ killing the cells and the ferment then digesting and separating them.

The ulcer begins as a tiny necrotic spot in the superficial mucous membrane, which separates. The necrosis spreads in all directions, and the tissue is gradually separated until an ulcer, involving the whole thickness of the mucous membrane, is produced, quite comparable to the acute spreading ulcer in man (Fig. 4).

The smallest ulcers are circular and as big as a pin's head. They often spread irregularly, but in the monkey referred to the ulcer spread in a regular shape to about half an inch long, lying parallel to and near the small curvature on the posterior wall of the stomach.

The strengths of acid necessary to produce this type of lesion render it unlikely that hyperacidity alone in man initiates it, unless the latter is of unusually high degree and combined with retention of food. If, however, a cellular poison is circulating in the blood, then a low degree of hyperacidity would be able to complete the death of the tissue, and the gastric juice would then form an ulcer.

Hyperacidity, particularly if combined with motor insufficiency, is therefore a factor of great importance, to be counted with both in the initiation and spread of an nlcer of the stomach.

Funnel Shape of Ulcer due to Gastric Juice.

Some time ago I gave my reasons for the belief that the funnel shape of a gastric ulcer is due to the action of the gastric juice. In the early stages of an ulcer which is extending laterally in the mucous membrane one can see that it has a shallow funnel shape with terraced edges due to destruction of the various layers of the gastric wall one after another. As time goes on, and the ulcer stops its spread, thickening gradually occurs round the edges and in the base by the formation of inflammatory tissue. The funnel shape becomes lost by elevation of the edges and undermining of the same by the action of the gastric juice. The subsequent shape varies greatly, owing to irregular lateral extensions at intervals, or to the base becoming adherent to some organ which is resistant to the action of the gastric juice. In other cases the funnel shape is more marked in the early stages owing to the limited superficial extent of the ulcer; digestion rapidly occurs in the base, and the ulcer perforates. Or the two processes of inflammatory thickening and digestion are more equally matched, and a funnel-shaped chronic ulcer results. The edges of this ulcer also become undermined and a more or less globular cavity results, the funnel shape being lost. All these changes are due to digestion of the base of the ulcer and to secondary inflammatory thickening largely owing to the necrotic and irritant actions of the hydrochloric acid.

Bacteria from the food infect the base to some extent, and so a very mild form of peritonitis leading to adhesions is established. Extensions in the mucous membrane, by which the ulcer becomes larger, are due to persistence or recrudescence of the original cause of the ulcer; but, how. ever the ulcer is originally produced, the connective tissue base is vulnerable to the action of the gastric juice, and is acted upon as described above.

\section{Want of Healing Power due to Hyperacidity} AND Motor InsUfFiciency.

I concluded that the unhealed condition was entirely due to the necrotic condition of the base, which is present in every chronic ulcer, the epithelium being unable to grow over it. The epithelium is quite able to proliferate, and is continually doing so even though advanced gastritis is present, but it can only grow over a base covered with granulation tissue, which is absent in chronic ulcer.

Clinical support is given to this view by the fact that the more chronic an ulcer is the more difficult and prolonged is the healing process owing to the changes in the base, and that healing is promoted by ensuring that the stomach empties itself in the normal time, and that hyperacidity is corrected by giving a diet which producess the minimum amount of secretion and by the administration of alkalis at the stage in the digestive process when free hydrochloric acid is present in the stomach. Rest in bed is also essential, because the diet can be more easily regulated, the irritability of the stomach reduced, and the dis orders of motion and secretion more easily corrected than when the patient is moving about. It is practically certain that every patient with chronic gastric ulcer is subject to attacks of motor and secretory disorders. My experiments also support the view that the failure of a chronic ulcer to heal is due to the condition of the base and not to a fault in the epithelium, and also that the prolonged action of the acid gastric juice, particularly when hyperacid, is perhaps the chief factor leading to this state.

At the outset I may state that, although by disordering the gastric functions one may definitely delay the healing of an experimentally produced ulcer for a considerable time, an actual arrest has not hitherto resulted. But, as I have already pointed out, the only methods available of producing hyperacidity and motor insufficiency are defective, so that the results I am going to describe should be considerably exaggerated before they are applied to the human subject.

In considering the question of variations in the healing of experimental ulcer I must first explain how the ulcers were produced and how they heal normally. The two animals that $I$ have employed in these investigations are the cat and the monkey. The ulcers were produced in each case by the local injection into the wall of the stomach of a gastrotoxic serum formed by immunizing the goat with the gastric cells of the cat or monkey.

An ulcer is thus produced very easily. It is necessary to open the abdomen, the stomach is drawn out, and the serum injected into any point under the peritoneum which may be selected.

The serum acts upon the cells with which it comes into contact, and these are then digested by the gastric juice, with the result that a cleanly punched-out ulcer is formed in a few days. The rate of formation is quicker in proportion to the degree of acidity of the gastric juice. The edge of the ulcer becomes smooth and rounded from the growth over its surface of epithelial cells derived from the adjacent glandular epithelium. From this edge the film of epithelium gradually grows over the base, which has now become covered with granulation tissue. In the meantime the base contracts, and the edges are brought together, sometimes to a remárkable extent considering the original size of the ulcer. As the base is continually becoming smaller there is an increasingly smaller surface to be covered with epithelium. This contraction of the base undoubtedly facilitates healing, because, if the wall of the stomach is stitched to the liver so tliat the base contracts with difficulty, the epithelium takes rather longer to cover the base. I have proved this in the case of the monkey. When the base is once covered with a layer of epithelium, and so protected from the gastric juice, the new mucous nembrane is formed from this quite regularly a few weeks later.

In order to estimate the rate of healing under various conditions one must select some stage in the healing process as the standard of comparison, and the best stage for this purpose is the point at which the base is just covered with a single layer of epithelial cells. The reason for this is that up to this point delay may occur; but when once the base is covered with cells there is no delay, and the mucous membrane always regularly re-forms to the best that it is capable of, and, further, it is a point which can be at once recognized with ease.

EFfects of Diet.

I found that the rate of healing depended upon the diet of the animal, or in other words upon the activity of the stomach with regard to secretion and motion.

This point was tested in the cat, owing to its suitability from a dietetic point of viow. After a full meal of cat's 
meat the animal's stomach is not empty for about twelve hours, whereas after a full meal of milk it is empty in about three hours. In the case of meat-fed animals, therefore, the base of a gastric ulcer is more continually exposed to the action of the hydrochloric acid of the gastric juice than in the case of milk-fed animals.

I found in a series of experiments that in the case of milk-fed animals the base of the ulcer was in most cases completely covered with a single layer of epithelial cells on the twentieth day and almost completely in a minority.

In the case of meat-fed cats this stage in the healing was reached at a later period, and in these cases the delay was caused by necrosis of the granulation tissue of the base of the ulcer so that the growth of the epithelium over it was delayed. Before healing could occur it was necessary for this superficial slough to separate.

In the case of milk-fed cats the superficial base of the ulcer was formed of granulation tissue at quite an early stage, but the prolonged action of the hydrochloric acid, assisted by the more vigorous movements of the stomach in meat-fed animals, killed off the superficial portions of this tissue, and by this means delayed the growth of the epithelium over it.

EFfects of Motor Insufficiency.

This same action of the gastric juice in delaying the healing of an ulcer can be more strikingly demonstrated by producing retention of food beyond the normal time and dilatation of the stomach by artificial pyloric stenosis. The duodenum of the cat was constricted by a short piece of rubber catheter tied on the outside. In a series of experiments of this nature I found that the healing of an ulcer was. delayed according to the degree of retention of food in the stomach. One can only proceed up to a certain degree of food retention, limited by the point beyond which it is impossible to maintain the normal digestive process, owing to vomiting or extreme dilatation of the stomach with wasting and death. The delay in cases of retention of food owing to motor insufficiency I found to be about twice the normal time, or even longer than this. The delay occurred in the early stages of healing and was due to necrosis of the granulation tissue base of the ulcer, so that the epithelium was unable to grow over it.

A further change was noted-namely, that the regeneration of the mucous membrane in these cases of delay did not occur in a normal manner. The glands consisted of cells of the duct type only and were very irregularly shaped and dilated. This latter change was due to the sclerotic condition of the base resulting from the chronic irritation of the hydrochloric acid, so that the glands had not a sufficiently vascular and cellular stroma in which to proliferate. A similar condition of newly-formed mucous membrane has for a long time been known to exist in healed gastric ulcers in the human subject.

EFfects of Hyperacidity.

So far we have been dealing with gastric juice of a normal acidity, but which has been allowed a longer time in which to act, and which has perhaps been assisted in its action by the increased peristalsis of the stomach.

We must now consider the effects of hyperacidity upon the rate of healing of an ulcer.

I had already done some experiments with guinea-pigs, the food being soaked in solutions of hydrochloric acid, but was dissatisfied with this method and the results. The following observations were made upon monkeys, the animals being fed daily by the tube with hydrochloric acid solution of different strengths. Any strength of hydro. chloric acid above the normal delays the healing of an ulcer, and the amount of delay varies with the strength of the solution.

Hydrochloric acid solution above 0.5 per cent. in strength is liable to be vomited, and perhaps largely for this reason stronger solutions do not produce a proportionately greater delay. Even a 1 per cent. solution either in the monkey or the cat will not cause extension of the ulcer, and the amount of delay in the healing is not much greater than with the weaker solution. In no case was the epithellum at the edge of the ulcer destroyed, giving rise to a regular lateral extension of the ulcer. If, however, the HCl was administered during the early stages of the formation of the alcer, the extent of the latter was much increased. The hydrochloric acid of the gastric juice is thus only able to cause lateral extension of an ulcer the origin of which is partially due to it; the lateral spread of other ulcers in the mucous membrane is due to the persistence of the original canses of the ulcers. In all the cases the edges of the ulcers were inverted and rounded by the superficial growth of new epithelium, and the delay in healing was due to necrosis of the granulation tissue of the base. In such cases the newly formed mucous membrane was of the atypical variety. The amount of delay in the healing was from two to three times the normal period (Fig. 5). On the vegetable diet which a monkey eats, an acute ulcer of the stomach heals in three weeks.

When motor insufficiency is combined with hyperacidity a summation of effects is observed. The motor insufficiency was produced in these monkeys by constricting the first part of the duodenum with silver wire twisted upon a glass rod, $4 \mathrm{~mm}$. in diameter, passed through a gastrotomy incision into the duodenum. An ulcer was also produced by injection of gastrotoxin. In three such monkeys, which were fed with strengths of $0.28,0.39$, and 0.9 per cent. hydrochloric acid solution respectively, large open ulcers were found on the twenty-first day. The bases were covered with necrotic tissue, and the epithelium had just rounded off the edges of the ulcers. All three were in very much the same condition, and their sizes were not much less than the original sizes of the ulcers (Fig. 6).

I have not observed the condition of such cases beyond the third week, but the presumption is that they would eventually heal. Another animal with a higher degree of pyloric obstruction and fed with 0.38 per cent. hydrochloric acid solution, died on the sixteenth day. A large excavated ulcer was found at the site of injection, and quite as big as the originally produced ulcer.

The delay in healing in all these cases must be ascribed to the necrotic action of the hydrochloric acid, and, as I have before remarked, in the human being the effect of hyperacidity must be considerably greater than in my experiments.

A similar condition of delayed healing of acute ulcer is found in human pathology. As a fact, one finds acute ulcers which heal readily, acute ulcers which are spreading, acute ulcers which are delayed in their healing, and definito chronic ulcers the healing of which is arrested and remains so for long periods of time.

In both the ulcers in which the healing is delayed and in chronic ulcer in which it is arrested, the same condition of the base is present-namely, necrosis of the connective tissue and secondary inflammatory thickening-and this is the reason why the ulcer is delayed or arrested in the healing. The fault is not in the epithelium, but in the connective tissue base of the ulcer. Motor insufficiency alone is able to delay the healing, and if one could efficiently produce hyperacidity even of a low degree in addition, it appears likely that a complete arrest in the healing of an experimental ulcer would be accomplished. We thus see what a very powerful factor the hydrochloric acid of the gastric juice is and what an important part it plays in the various pathological processes which constitute the life-history of a gastric ulcer.

\section{ON GASTRIC ANALYSIS.}

BY

TREVOR HEATON, M.B.OxoN., M.R.C.P., MEDICAL REGISTRAR, GUY'S HOSPITAL.

THE analysis of the contents of the stomach after a test meal must always be an inexact method of achieving its object-namely, the measurement of the amount and composition of the gastric secretion. The limitations of the method are obvious. Its value must be only in so far as it gives information which may be compared with similar data, obtained under similar conditions from patients who have been found, whether at operation or post nortem, to present the various phenomena of gastric pathology. The value of the method must therefore obviously be the greater the more varied this information can be made; and the more perfectly the conditions of obtaining it can be standardized. 


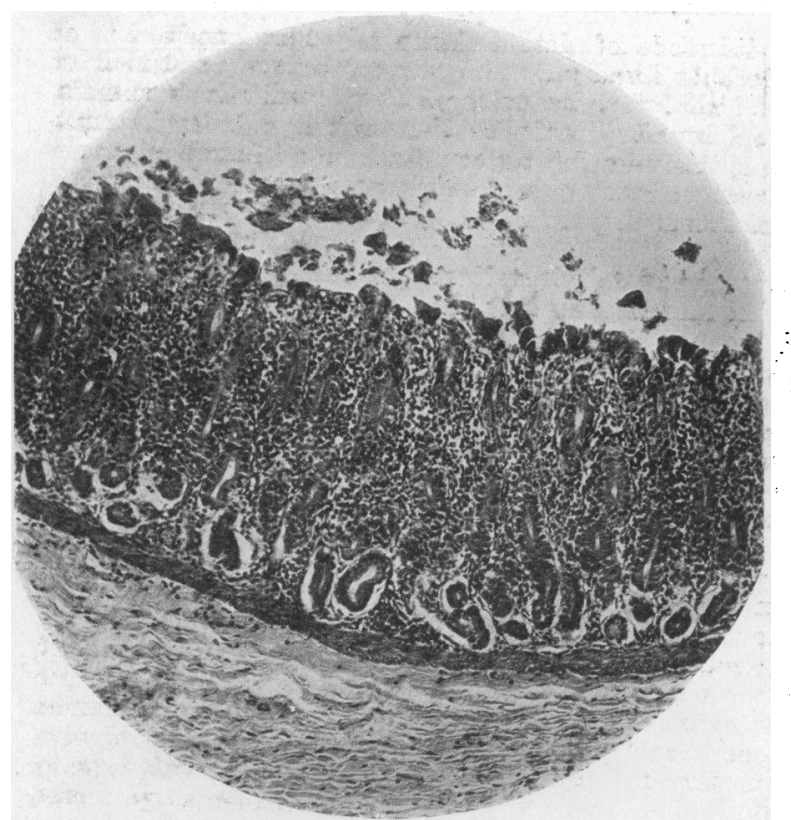

FIG. 1.

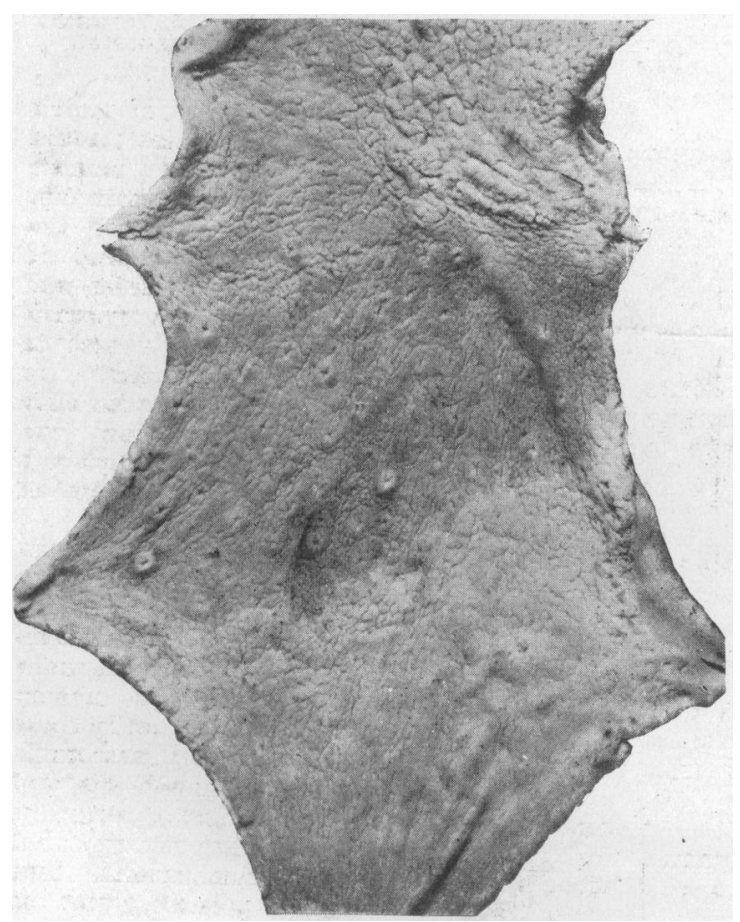

FIG. 3.

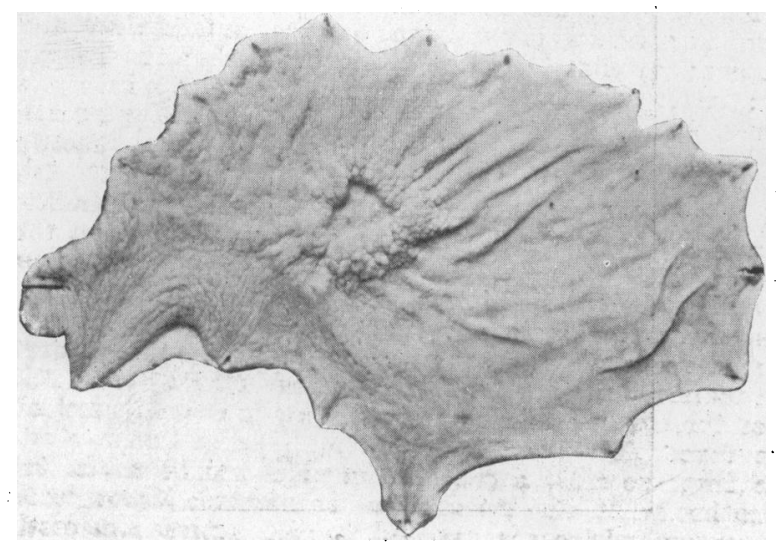

FIG. 5.

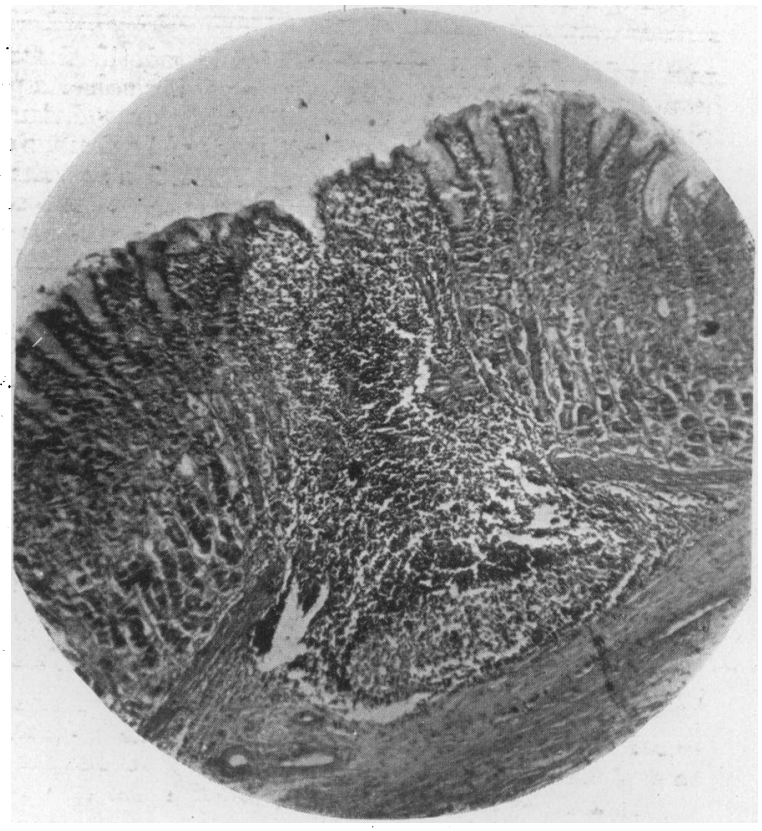

FIG. 2.

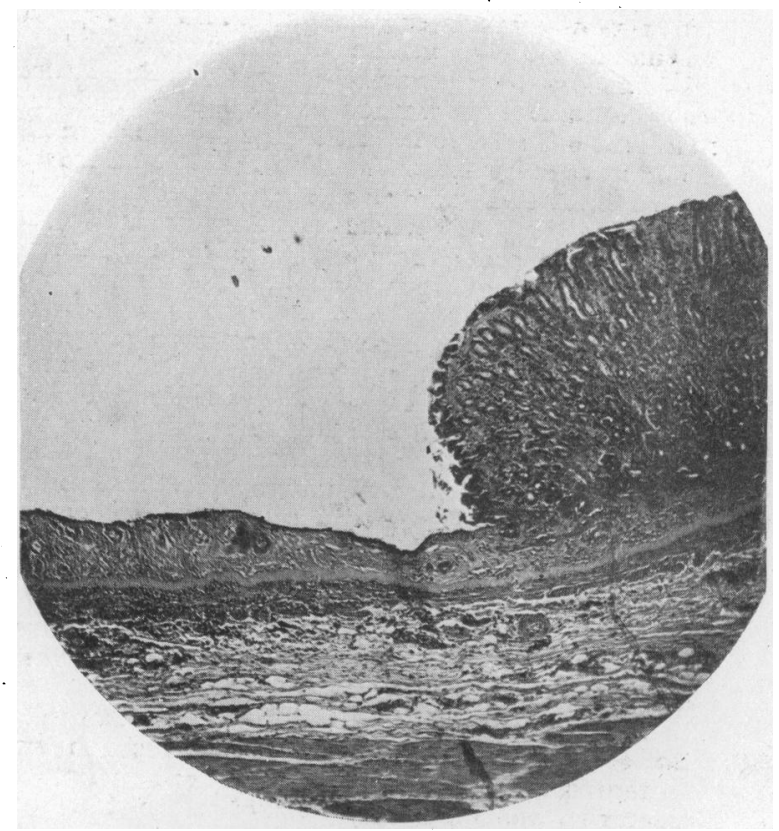

FIG. 4.

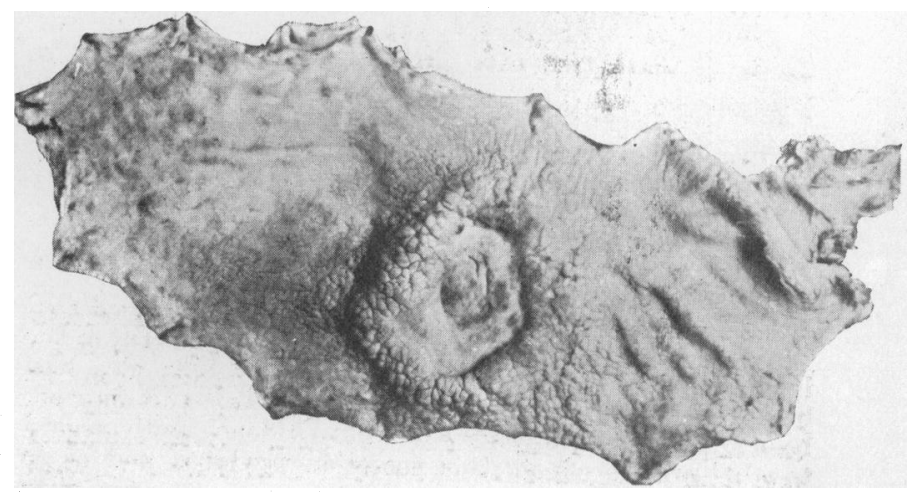

FIG. 6. 